\title{
Measuring $\boldsymbol{\beta}^{*}$ in SuperKEKB with $K$ modulation
}

\author{
P. Thrane $\odot,{ }^{1,2}$ R. Tomás $\odot,{ }^{2}$ A. Koval,${ }^{3,2}$ K. Ohmi,${ }^{4}$ Y. Ohnishi,${ }^{4}$ and A. Wegscheider ${ }^{5,2}$ \\ ${ }^{1}$ Norwegian University of Science and Technology, N-7491 Trondheim, Norway \\ ${ }^{2}$ CERN, Geneva CH-1211, Switzerland \\ ${ }^{3}$ University of Dundee, Nethergate, Dundee, DD1 4HN, Scotland, United Kingdom \\ ${ }^{4}$ KEK, 1-1 Oho, Tsukuba, Ibaraki 305-0801, Japan \\ ${ }^{5}$ Universität Hamburg, Mittelweg 177, 20148 Hamburg, Germany
}

(Received 29 March 2019; published 28 January 2020)

\begin{abstract}
SuperKEKB is an asymmetric energy electron positrion collider currently under commissioning in Japan. It aims to achieve a record high luminosity of $8 \times 10^{35} \mathrm{~cm}^{-2} \mathrm{~s}^{-1}$, for which accurate control of $\beta_{y}^{*}$ is needed. The advanced final focus system is also relevant for studies related to future linear colliders, which similarly need to achieve very small beam sizes. To work for SuperKEKB, the $K$ modulation technique is generalized to allow known quadrupole fields between the modulated magnets and the interaction point. Initial measurements taken in HER agree with simulations, and show that $K$ modulation is suitable for measuring the minimum of the $\beta$ function at the interaction point within $1 \%$, however it is too uncertain to be used for measuring the displacement of the beam waist away from the interaction point. In addition, tune shift equations for a modulated quadrupole are derived without assuming a thin lens perturbation, giving a simple method to calculate the tune shift to second order in the quadrupole strength modulation.
\end{abstract}

DOI: 10.1103/PhysRevAccelBeams.23.012803

\section{INTRODUCTION}

This article is based on results available in [1]. Currently under commissioning, SuperKEKB is an asymmetric energy electron positron collider at the High Energy Accelerator Research Organization in Japan (KEK) [2]. It is composed of a $7 \mathrm{GeV}$ ring for electrons and a $4 \mathrm{GeV}$ ring for positrons, called the High Energy Ring (HER) and Low Energy Ring (LER), respectively. The accelerator is an upgraded version of KEKB which operated between 1999 and 2010, during which the physics experiment Belle collected an integrated luminosity of $1.04 \mathrm{ab}^{-1}$, and achieved a record high instantaneous luminosity of $2.11 \times 10^{34} \mathrm{~cm}^{-2} \mathrm{~s}^{-1}$ in 2009 [3]. This made possible the detection of $C P$ violation in $B$ mesons and contributed to the confirmation of the Kobayashi-Maskawa theory [4]. SuperKEKB and the upgraded Belle II aim at a luminosity of $8 \times 10^{35} \mathrm{~cm}^{-2} \mathrm{~s}^{-1}$, surpassing the previous record by a factor 40. This will allow for an integrated luminosity of $50 \mathrm{ab}^{-1}$, enabling precision measurements of rare events and thus testing of the Standard Model as well as potentially revealing hints about new physics [5].

Published by the American Physical Society under the terms of the Creative Commons Attribution 4.0 International license. Further distribution of this work must maintain attribution to the author(s) and the published article's title, journal citation, and DOI.
This factor 40 increase in luminosity comes mainly from two improvements: increasing the beam current by a factor of 2 and decreasing $\beta_{y}^{*}$ by a factor 20 down to $0.3 \mathrm{~mm}, \beta_{y}^{*}$ being the vertical $\beta$ function at the interaction point (IP). See Table I for a list of machine parameters. The luminosity is inversely proportional to $\beta_{y}^{*}$, and precise measurements along with control of this value is therefore important. Several well established techniques exist for measuring the $\beta$ function, and an overview including comparisons between the methods is found in [6]. Currently in SuperKEKB, $\beta$ function measurements are done globally using an orbit response matrix method [7]. $K$ modulation directly measures the average $\beta$ function at a modulated quadrupole by measuring the corresponding tune shift. As a result $K$ modulation is slow for global measurements, but can be used for measuring $\beta^{*}$ and has been used to do so in other machines with uncertainty below $1 \%[8,9]$. However, it has been shown that the uncertainty of $\beta^{*}$ measurements using $K$ modulation grows significantly for small values of $\beta^{*}[10]$, raising the question whether $K$ modulation will give accurate measurements in SuperKEKB.

Precise $\beta^{*}$ measurements in SuperKEKB are interesting for other machines as well. Future linear colliders like CLIC [11] and ILC [12] require luminosities on the order of $10^{34} \mathrm{~cm}^{-2} \mathrm{~s}^{-1}$ to meet the demands of the experiments. Such luminosities further require small beam sizes and strong focusing quadrupole magnets with large chromatic aberrations that must be corrected in the final focus 
TABLE I. Main parameters of SuperKEKB [2].

\begin{tabular}{|c|c|c|c|c|}
\hline & & $\operatorname{LER}\left(e^{+}\right)$ & $\operatorname{HER}\left(e^{-}\right)$ & Unit \\
\hline Energy & $E$ & 4.000 & 7.007 & $\mathrm{GeV}$ \\
\hline Current & $I$ & 3.6 & 2.6 & A \\
\hline Number of bunches & \multicolumn{4}{|c|}{2500} \\
\hline Bunch current & & 1.44 & 1.04 & $\mathrm{~mA}$ \\
\hline Circumference & $C$ & \multicolumn{2}{|c|}{3016.315} & $\mathrm{~m}$ \\
\hline Emittance & $\epsilon_{x} / \epsilon_{y}$ & $3.2 / 8.64$ & $4.6 / 12.9$ & $\mathrm{~nm} / \mathrm{pm}$ \\
\hline Coupling & & 0.27 & 0.28 & $\%$ \\
\hline$\beta$ function at IP & $\beta_{x}^{*} / \beta_{y}^{*}$ & $32 / 0.27$ & $25 / 0.30$ & $\mathrm{~mm}$ \\
\hline Transverse beam size at IP & $\sigma_{x}^{*} / \sigma_{y}^{*}$ & $10.1 / 48$ & $10.7 / 62$ & $\mu \mathrm{m} / \mathrm{nm}$ \\
\hline Crossing angle & & \multicolumn{2}{|c|}{83} & $\operatorname{mrad}$ \\
\hline Momentum compaction & $\alpha_{p}$ & 3.20 & 4.55 & $10^{-4}$ \\
\hline Energy spread & $\sigma_{\delta}$ & 7.92 & 6.37 & $10^{-4}$ \\
\hline Total cavity voltage & $V_{c}$ & 9.4 & 15.0 & MV \\
\hline Bunch length & $\sigma_{z}$ & 6.0 & 5.0 & $\mathrm{~mm}$ \\
\hline Synchrotron tune & $\nu_{s}$ & 0.0245 & 0.0280 & \\
\hline Betatron tune & $Q_{x} / Q_{y}$ & $44.53 / 46.57$ & $45.53 / 43.57$ & \\
\hline Energy loss per turn & $U_{0}$ & 1.76 & 2.43 & $\mathrm{MeV}$ \\
\hline Damping time & $\tau_{x, y} / \tau_{z}$ & $43.2 / 22.8$ & $58.0 / 29.0$ & msec \\
\hline Beam-beam parameter & $\xi_{x} / \xi_{y}$ & $0.0028 / 0.0881$ & $0.0012 / 0.807$ & \\
\hline Luminosity & $\mathrm{L}$ & \multicolumn{2}{|c|}{$8 \times 10^{35}$} & $\mathrm{~cm}^{-2} \mathrm{~s}^{-1}$ \\
\hline
\end{tabular}

systems. For CLIC there are currently two types of focusing systems proposed [13], one of which is also the baseline for ILC and is being tested at ATF2 [14]. The other focusing system is similar to the one used to correct the chromaticity from the final focusing quadrupoles in SuperKEKB LER and HER. This focusing system was tested at FFTB [15], but SuperKEKB will demonstrate the system at smaller IP beam sizes and initial studies have investigated the possibility of reducing $\beta_{y}^{*}$ by a factor 3 beyond the nominal value [16], which would give a further $40 \%$ reduction in the vertical beam size at the IP if chromaticity and nonlinear effects can be properly corrected. Such dedicated studies of the final focus system would make it possible to compare
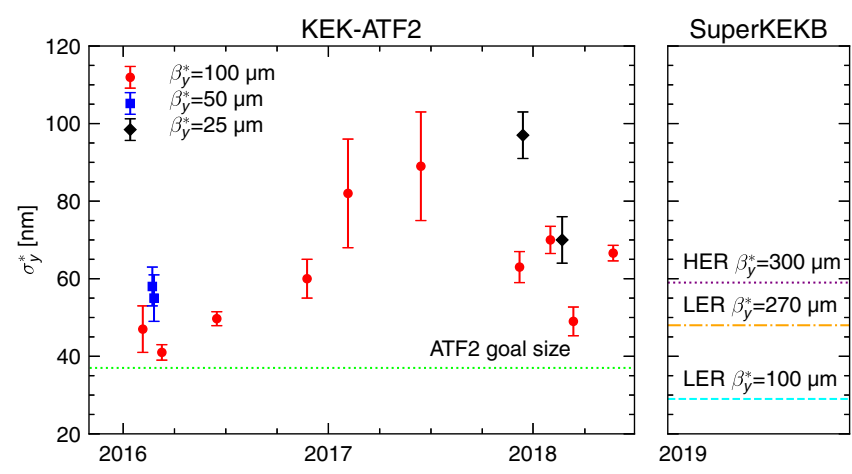

FIG. 1. Measured vertical beam sizes in ATF2 [17] for different values of $\beta_{x}^{*}$ and $\beta_{y}^{*}$ as compared to the nominal value. On the left are the nominal vertical beam sizes intended for SuperKEKB rings HER and LER. An additional line is added for the case of reduced $\beta_{y}^{*}$ in LER. the SuperKEKB system with that used in ATF2, and would require accurate $\beta^{*}$ measurements. Figure 1 shows vertical beam size measurements from ATF2, together with the planned beam size for HER and LER, as well as a tentative goal for a possible focus system study. Table II displays the chromaticity of the various machines discussed above, approximated using the formula $\xi_{y} \sim \mathrm{L}^{*} / \beta_{y}^{*}$.

\section{THEORY AND METHOD}

\section{A. Tune shift from modulation of a quadrupole magnet}

We seek an expression for the change in tune when slightly changing the strength of a quadrupole magnet. This is normally done by assuming the perturbation can be approximated as a thin quadrupole, following the method described in [18]. By acting on the one turn matrix $\boldsymbol{T}_{\text {original }}$ with a thin lens matrix we get the new full turn transfer matrix $\boldsymbol{T}_{\text {new }}$

TABLE II. Comparison of chromaticity, $\xi_{y} \sim\left(\mathrm{L}^{*} / \beta_{y}^{*}\right)$, in the final focusing quadrupoles for CLIC, ILC, ATF2, FFTB and the Low/High Energy Ring in SuperKEKB.

\begin{tabular}{lccc}
\hline \hline & $\mathrm{L}^{*}[\mathrm{~m}]$ & $\beta_{y}^{*}[\mu \mathrm{m}]$ & $\mathrm{L}^{*} / \beta_{y}^{*}$ \\
\hline CLIC & 3.5 & 70 & 50000 \\
ILC & $3.5 / 4.1$ & 410 & $8500 / 10000$ \\
ATF2 & 1 & 100 & 10000 \\
FFTB & 0.4 & 100 & 4000 \\
SuperKEKB LER & 0.94 & 270 & 3500 \\
SuperKEKB HER & 1.41 & 300 & 4700 \\
\hline \hline
\end{tabular}




$$
\boldsymbol{T}_{\text {new }}=\boldsymbol{T}_{\text {original }}\left(\begin{array}{cc}
1 & 0 \\
\pm \Delta K L & 1
\end{array}\right)
$$

where $\Delta K L$ is the change in integrated strength of the magnet. The two transfer matrices are given by

$$
\begin{aligned}
& \boldsymbol{T}_{\text {original }} \\
& =\left(\begin{array}{cc}
\cos (2 \pi Q)+\alpha_{0} \sin (2 \pi Q) & \beta_{0} \sin (2 \pi Q) \\
-\gamma_{0} \sin (2 \pi Q) & \cos (2 \pi Q)-\alpha_{0} \sin (2 \pi Q)
\end{array}\right),
\end{aligned}
$$

$$
\begin{aligned}
& \boldsymbol{T}_{\text {new }} \\
& =\left(\begin{array}{cc}
\cos \left(2 \pi Q^{\prime}\right)+\alpha_{0}^{\prime} \sin \left(2 \pi Q^{\prime}\right) & \beta_{0}^{\prime} \sin \left(2 \pi Q^{\prime}\right) \\
-\gamma_{0}^{\prime} \sin \left(2 \pi Q^{\prime}\right) & \cos \left(2 \pi Q^{\prime}\right)-\alpha_{0}^{\prime} \sin \left(2 \pi Q^{\prime}\right)
\end{array}\right) .
\end{aligned}
$$

The new tune $Q^{\prime}$ is equal to the original tune $Q$ plus the tune shift $\Delta Q \cdot \beta_{0}, \alpha_{0}$, and $\gamma_{0}$ are the optical functions at the thin lens quadrupole before the modulation, while $\beta_{0}^{\prime}, \alpha_{0}^{\prime}$, and $\gamma_{0}^{\prime}$ are the optical functions after the modulation. See Fig. 2 for an illustration of the method. By taking the trace of both sides of Eq. (1) we get

$$
2 \cos (2 \pi Q) \pm \beta_{0} \Delta K L \sin (2 \pi Q)=2 \cos [2 \pi(Q+\Delta Q)]
$$

This equation can also be used also for a thick quadrupole by approximating $\beta_{0} \approx \bar{\beta}$.

In the above, the quadrupole modulation was approximated with a thin quadrupole. We show now a slightly different approach that reproduces the same results to linear

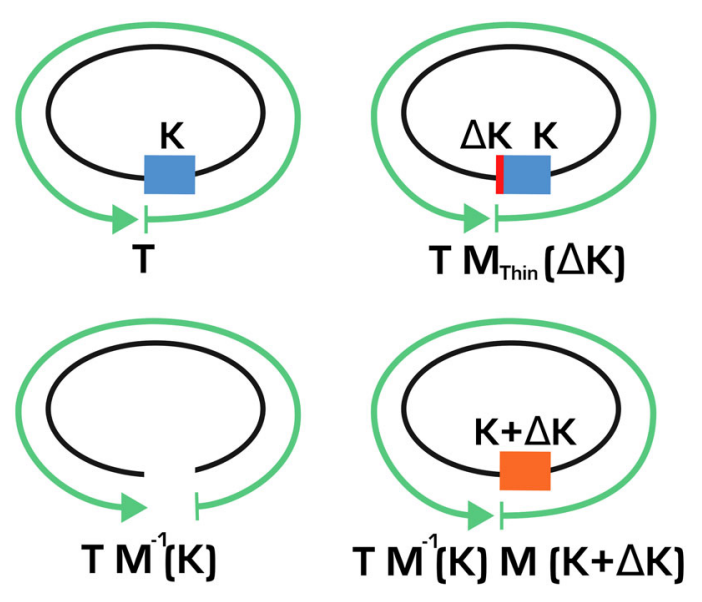

FIG. 2. Illustration of a one turn matrix (top left), and a one turn matrix with a thin quadrupole perturbation $\Delta K$ (top right). Below is a one turn matrix where a quadrupole of strength $K$ is removed (bottom left), and a one turn matrix where a quadrupole of strength $K$ has been replaced by a quadrupole of strength $K+\Delta K$ (bottom right). order in $\Delta K$, but that also gives the second order tune shift from $\Delta K$ without assuming a thin quadrupole. Instead of using a thin lens perturbation, we replace the magnet of strength $K$ with a magnet of strength $K+\Delta K$ by acting on the one turn matrix with the inverse of the original quadrupole transfer matrix, $\boldsymbol{M}^{-1}(K)$, followed by the transfer matrix of the modulated quadrupole $\boldsymbol{M}(K+\Delta K)$, see Fig. 2. This gives the one turn transfer matrix $\boldsymbol{T}_{\text {new }}$ including the modulation

$$
\boldsymbol{T}_{\text {new }}=\boldsymbol{T}_{\text {original }} \boldsymbol{M}^{-1}(K) \boldsymbol{M}(K+\Delta K) .
$$

For a focusing quadrupole these matrices are given by

$$
\begin{gathered}
\boldsymbol{M}(K)=\left(\begin{array}{cc}
\cos (\sqrt{K} L) & \frac{\sin (\sqrt{K} L)}{\sqrt{K}} \\
-\sqrt{K} \sin (\sqrt{K} L) & \cos (\sqrt{K} L)
\end{array}\right), \\
\boldsymbol{M}^{-1}(K)=\left(\begin{array}{cc}
\cos (\sqrt{K} L) & -\frac{\sin (\sqrt{K} L)}{\sqrt{K}} \\
\sqrt{K} \sin (\sqrt{K} L) & \cos (\sqrt{K} L)
\end{array}\right) .
\end{gathered}
$$

Calculating $\boldsymbol{M}^{-1}(K) \boldsymbol{M}(K+\Delta K)$ and expanding to second order in $\Delta K$ gives

$$
\begin{aligned}
\boldsymbol{M}^{-1} & (K) \boldsymbol{M}(K+\Delta K) \\
& =\left(\begin{array}{cc}
1+a_{11} \Delta K+b_{11} \Delta K^{2} & a_{12} \Delta K+b_{12} \Delta K^{2} \\
a_{21} \Delta K+b_{21} \Delta K^{2} & 1+a_{22} \Delta K+b_{22} \Delta K^{2}
\end{array}\right) \\
& =\boldsymbol{I}+\boldsymbol{a} \Delta K+\boldsymbol{b} \Delta K^{2},
\end{aligned}
$$

where the coefficients $a_{i j}$ and $b_{i j}$ are given by, writing $\sqrt{K} L=\phi$,

$$
\begin{gathered}
a_{11}=\frac{\sin ^{2}(\phi)}{2 K}, \\
a_{12}=\frac{L}{2 K}\left(1-\frac{\sin (2 \phi)}{2 \phi}\right), \\
a_{21}=-\frac{L}{2}\left(1+\frac{\sin (2 \phi)}{2 \phi}\right), \\
b_{11}=\frac{1}{8 K^{2}}\left[\phi \sin (2 \phi)-\phi^{2}-\sin ^{2}(\phi)\right], \\
b_{12}=\frac{1}{16 K^{5 / 2}}\left[3 \sin (2 \phi)-2 \phi-4 \phi \cos ^{2}(\phi)\right], \\
b_{21}=\frac{1}{16 K^{3 / 2}}\left[\sin (2 \phi)+2 \phi-4 \phi \cos ^{2}(\phi)\right], \\
b_{22}=\frac{1}{8 K^{2}}\left[-\phi \sin (2 \phi)-\phi^{2}+3 \sin ^{2}(\phi)\right] .
\end{gathered}
$$


For completeness, the coefficients for a defocusing quadrupole are found the same way and are given by

$$
\begin{gathered}
a_{11, \mathrm{def}}=-\frac{\sinh ^{2}(\phi)}{2 K}, \\
a_{12, \mathrm{def}}=\frac{L}{2 K}\left(1-\frac{\sinh (2 \phi)}{2 \phi}\right), \\
a_{21, \mathrm{def}}=\frac{L}{2}\left(1+\frac{\sinh (2 \phi)}{2 \phi}\right), \\
a_{22, \mathrm{def}}=\frac{\sinh ^{2}(\phi)}{2 K}=-a_{11, \mathrm{def}},
\end{gathered}
$$

$$
\begin{gathered}
b_{11, \mathrm{def}}=-\frac{1}{8 K^{2}}\left[\phi \sinh (2 \phi)-\phi^{2}-\sinh ^{2}(\phi)\right], \\
b_{12, \mathrm{def}}=\frac{1}{16 K^{5 / 2}}\left[3 \sinh (2 \phi)-2 \phi-4 \phi \cosh ^{2}(\phi)\right], \\
b_{21, \mathrm{def}}=-\frac{1}{16 K^{3 / 2}}\left[\sinh (2 \phi)+2 \phi-4 \phi \cosh ^{2}(\phi)\right], \\
b_{22, \mathrm{def}}=-\frac{1}{8 K^{2}}\left[-\phi \sinh (2 \phi)-\phi^{2}+3 \sinh ^{2}(\phi)\right] .
\end{gathered}
$$

Using Eq. (6) we can then find an expression for the trace of $\boldsymbol{T}_{\text {new }}$ in Eq. (4)

$$
\begin{aligned}
\operatorname{Tr}\left(\boldsymbol{T}_{\text {new }}\right)= & \cos (2 \pi Q)\left[2+\left(a_{11}+a_{22}\right) \Delta K+\left(b_{11}+b_{22}\right) \Delta K^{2}\right]+\alpha_{0} \sin (2 \pi Q)\left[\left(a_{11}-a_{22}\right) \Delta K+\left(b_{11}-b_{22}\right) \Delta K^{2}\right] \\
& +\beta_{0} \sin (2 \pi Q)\left(a_{21} \Delta K+b_{21} \Delta K^{2}\right)+\gamma_{0} \sin (2 \pi Q)\left(-a_{12} \Delta K-b_{12} \Delta K^{2}\right),
\end{aligned}
$$

where $\beta_{0}, \alpha_{0}$ and $\gamma_{0}$ are the optical functions at the edge of the modulated magnet. Note that $a_{11}=-a_{22}$ such that $a_{11}+a_{22}=0$ in Eq. (9). We know that the trace of $\boldsymbol{T}_{\text {new }}$ is also equal to $2 \cos (2 \pi(Q+\Delta Q))$. To linear order in $\Delta K$ we insert the coefficients $a_{11}, a_{12}, a_{21}, a_{22}$ and solve

$$
2 \cos [2 \pi(Q+\Delta Q)]=2 \cos (2 \pi Q)-\bar{\beta} \Delta K L \sin (2 \pi Q),
$$

where the average $\beta$ function in a focusing hard edge quadrupole is given by [10]

$$
\bar{\beta}=\beta_{0} u_{0} \pm \alpha_{0} u_{1}+\gamma_{0} u_{2}
$$

and the sign depends on if the quadrupole is upstream or downstream of the IP. For a focusing and defocusing magnet the coefficients are respectively

$$
\begin{gathered}
u_{0, \mathrm{foc}}=\frac{1}{2}\left(1+\frac{\sin (2 \phi)}{2 \phi}\right)=-\frac{a_{21}}{L}, \\
u_{1, \mathrm{foc}}=\frac{\sin ^{2}(\phi)}{K L}=\frac{a_{11}-a_{22}}{L}, \\
u_{2, \mathrm{foc}}=\frac{1}{2 K}\left(1-\frac{\sin (2 \phi)}{2 \phi}\right)=\frac{a_{12}}{L}, \\
u_{0, \mathrm{def}}=\frac{1}{2}\left(1+\frac{\sinh (2 \phi)}{2 \phi}\right)=\frac{a_{21, \mathrm{def}}}{L}, \\
u_{1, \mathrm{def}}=\frac{\sinh ^{2}(\phi)}{K L}=-\frac{a_{11, \mathrm{def}}-a_{22, \mathrm{def}}}{L}, \\
u_{2, \mathrm{def}}=-\frac{1}{2 K}\left(1-\frac{\sinh (2 \phi)}{2 \phi}\right)=-\frac{a_{12, \mathrm{def}}}{L} .
\end{gathered}
$$

Equation (10) is exactly equal to Eq. (3), when assuming $\beta_{0} \approx \bar{\beta}$, but now we have included the length of the modulated quadrupole in the calculation. For a defocusing quadrupole the only difference is a change of sign as included in Eq. (3).

Returning to Eq. (9), we can now find the change to Eq. (10) due to the second order terms in $\Delta K$

$2 \cos [2 \pi(Q+\Delta Q)]=2 \cos (2 \pi Q)-\bar{\beta} \Delta K L \sin (2 \pi Q) D$.

With $D$ equal to

$$
\begin{gathered}
D=(1-\Delta K E), \\
E=\frac{\cot (2 \pi Q)\left(b_{11}+b_{22}\right)+b_{21} \beta_{0}+\left(b_{11}-b_{22}\right) \alpha_{0}-b_{12} \gamma_{0}}{a_{21} \beta_{0}+\left(a_{11}-a_{22}\right) \alpha_{0}-a_{12} \gamma_{0}} .
\end{gathered}
$$

Assuming the edge of the quadrupole is separated from the IP by a drift of length $L^{*}$, we can propagate the optical functions in the drift from the waist to the edge of the magnet, giving

$$
\begin{gathered}
D=(1-\Delta K E), \\
E=\frac{\beta^{*} \cot (2 \pi Q)\left(b_{11}+b_{22}\right)+b_{21} L^{* 2}-\left(b_{11}-b_{22}\right) L^{*}-b_{12}}{a_{21} L^{* 2}-\left(a_{11}-a_{22}\right) L^{*}-a_{12}} .
\end{gathered}
$$

This equation can be used to estimate the error in Eq. (3) due to the second order terms of $\Delta K$ by inserting the relevant parameter values $\beta^{*}, L^{*}, Q, K$, and $L$. 
By inserting the design parameters for LER and HER in Eq. (15) we find an error of the order $10^{-6}$ in $\bar{\beta}$, which corresponds to a change in tune of less than $10^{-6}$, smaller than the uncertainty of the tune measurement. Note that it is possible to include the second order terms in the $K$ modulation measurement by using the coefficients from Eq. (9) instead of using $\bar{\beta}$ as done here and in [10], however the second order correction is small.

\section{B. $K$ modulation}

Modulating the strength of a quadrupole will give a tune shift as shown above. The orbit at the modulated quadrupole needs to be sufficiently small so that no significant orbit distortions are generated. During the experiment this is guaranteed by monitoring the closed orbit change. Measuring the tune shift and knowing the modulation strength thus gives a direct measurement of the $\bar{\beta}$ in the modulated quadrupole. By modulating the two quadrupoles closest to the IP and propagating the measured $\beta$ function it is possible to calculate $\beta^{*}$. This method has been derived in [10] and is presented below.

Propagating the optical functions from the IP through the drift length $L^{*}$, the optical functions at the edge of the last quadrupole before the IP are given by

$$
\begin{gathered}
\beta_{0}=\beta_{w}+\frac{\left(L^{*} \pm w\right)^{2}}{\beta_{w}}, \\
\alpha_{0}= \pm \frac{\left(L^{*} \pm w\right)}{\beta_{w}} \\
\gamma_{0}=\frac{1}{\beta_{w}}
\end{gathered}
$$

With $\beta=\beta_{w}$ at the waist of the beam, and the waist shifted a distance $w$ downstream of the IP, see Fig. 3 . The signs depend on whether the magnet is upstream or downstream of the IP. Here and in the following, the upper sign will denote the case where the magnet is upstream, and the lower sign is for the downstream magnet. The average $\beta$ function in the quadrupole can be expressed by Eq. (11).

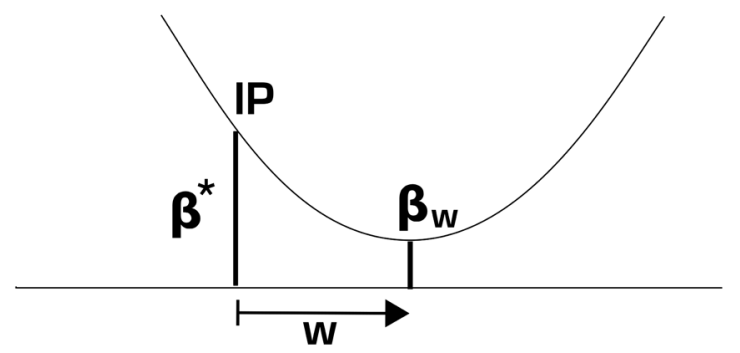

FIG. 3. The minimum of the $\beta$ function is $\beta_{w}$ and it is displaced longitudinally away from the IP a distance $w$. The $\beta$ function grows quadratically away from the waist. $w$ is defined as positive when the waist lies downstream from the IP.
Inserting the optical functions from Eq. (16) and assuming $\beta^{*} \ll L^{*}$ yields

$$
\bar{\beta}=\frac{\left(L^{*} \pm w\right)^{2}}{\beta_{w}} u_{0}+\frac{\left(L^{*} \pm w\right)}{\beta_{w}} u_{1}+\frac{1}{\beta_{w}} u_{2},
$$

which can be rewritten

$$
\beta_{w}=\frac{1}{\bar{\beta}}\left[\left(L^{*} \pm w\right)^{2} u_{0}+\left(L^{*} \pm w\right) u_{1}+u_{2}\right] .
$$

Equating Eq. (18) for the magnet upstream and downstream results in

$\frac{\bar{\beta}_{\text {upstream }}}{\bar{\beta}_{\text {downstream }}}=\frac{\left(L^{*}+w\right)^{2} v_{0}+\left(L^{*}+w\right) v_{1}+v_{2}}{\left(L^{*}-w\right)^{2} u_{0}+\left(L^{*}-w\right) u_{1}+u_{2}}=\chi$,

where the ratio between the average $\beta$ function in the upstream and downstream quadrupole is defined as $\chi$, which is close to 1 as the focusing system in SuperKEKB is symmetric around the IP. Note however that there is a slight asymmetry in the position and length of the horizontally focusing quadrupoles in HER, see Fig. 5. The coefficients $u_{i}$ for the upstream magnet have been changed to $v_{i}$ to distinguish them from those for the downstream magnet. Further rewriting leads to a second order equation for $w$

$$
\begin{aligned}
& \left(v_{0}-\chi u_{0}\right) w^{2}+\left(2 h v_{0}+v_{1}+2 \chi h u_{0}+\chi u_{1}\right) w \\
& \quad+\left(h^{2} v_{0}+h v_{1}+v_{2}-\chi h^{2} u_{0}-\chi h u_{1}-\chi u_{2}\right)=0
\end{aligned}
$$

where $h=L^{*}$ is introduced here for convenience later. Solving for $w$, Eq. (18) can be used to solve $\beta_{w}$ for each magnet. Taking the average of these $\beta_{w}, \beta^{*}$ can then be found by again propagating from the waist

$$
\beta^{*}=\beta_{w}+\frac{w^{2}}{\beta_{w}} .
$$

\section{C. $K$ modulation with transfer matrices}

If there are quadrupole fields affecting the optical functions in between the final focusing quadrupole and the IP, the method in [10] cannot be used directly. This is the case for HER in SuperKEKB (Fig. 5). However, the method may be modified to include the effects of these fields by propagating the optical functions past them using a transfer matrix, assuming the fields are known. Let the optical functions at the end of the quadrupole magnet be given by $\beta_{1}, \alpha_{1}$ and $\gamma_{1}$. As before, the average $\beta$ function in the quadrupole is given by

$$
\bar{\beta}=\beta_{1} u_{0} \pm \alpha_{1} u_{1}+\gamma_{1} u_{2},
$$

To include the extra fields between the quadrupole and the IP, a point is chosen that lies between the fields and the IP. 
The point is separated from the IP by a drift of length $h$, and the optical functions at this point are $\beta_{0}, \alpha_{0}$, and $\gamma_{0}$. If we write the transfer matrix between this point and the edge of the magnet as

$$
\left(\begin{array}{ll}
C & S \\
C^{\prime} & S^{\prime}
\end{array}\right)
$$

the average $\beta$ function in the magnet can be expressed by $\beta_{0}, \alpha_{0}$, and $\gamma_{0}$

$$
\begin{aligned}
\bar{\beta}= & \beta_{1} u_{0} \pm \alpha_{1} u_{1}+\gamma_{1} u_{2} \\
= & u_{0}\left(C^{2} \beta_{0}-2 C S \alpha_{0}+S^{2} \gamma_{0}\right) \\
& \pm u_{1}\left(-C C^{\prime} \beta_{0}+\left(C S^{\prime}+S C^{\prime}\right) \alpha_{0}-S S^{\prime} \gamma_{0}\right) \\
& +u_{2}\left(C^{\prime 2} \beta_{0}-2 C^{\prime} S^{\prime} \alpha_{0}+S^{\prime 2} \gamma_{0}\right) \\
= & \beta_{0}\left(C^{2} u_{0} \mp C C^{\prime} u_{1}+C^{\prime 2} u_{2}\right) \\
& \pm \alpha_{0}\left(\mp 2 C S u_{0}+\left(C S^{\prime}+S C^{\prime}\right) u_{1} \mp 2 C^{\prime} S^{\prime} u_{2}\right) \\
& +\gamma_{0}\left(S^{2} u_{0} \mp S S^{\prime} u_{1}+S^{\prime 2} u_{2}\right) \\
= & \beta_{0} U_{0} \pm \alpha_{0} U_{1}+\gamma_{0} U_{2} .
\end{aligned}
$$

Note that the transfer matrix including $C, C^{\prime}, S$, and $S^{\prime}$ is defined as going from the point closest to the IP and until the magnet edge, regardless of whether the magnet is up- or downstream of the IP. Here the coefficients $U_{0}, U_{1}$, and $U_{2}$ do depend on whether the magnet is up- or downstream of the IP, and are given by

$$
\begin{gathered}
U_{0}=C^{2} u_{0} \mp C C^{\prime} u_{1}+C^{\prime 2} u_{2}, \\
U_{1}=\mp 2 C S u_{0}+\left(C S^{\prime}+S C^{\prime}\right) u_{1} \mp 2 C^{\prime} S^{\prime} u_{2}, \\
U_{2}=S^{2} u_{0} \mp S S^{\prime} u_{1}+S^{\prime 2} u_{2} .
\end{gathered}
$$

Propagating the optical functions from the beam waist near the IP, a distance $h$ away, we get

$$
\begin{gathered}
\beta_{0}=\beta_{w}+\frac{(h \pm w)^{2}}{\beta_{w}}, \\
\alpha_{0}= \pm \frac{(h \pm w)}{\beta_{w}}, \\
\gamma_{0}=\frac{1}{\beta_{w}} .
\end{gathered}
$$

With the assumption $\beta^{*} \ll h$, Eqs. (24) and (26) result in

$$
\beta_{w}=\frac{1}{\bar{\beta}}\left[(h \pm w)^{2} U_{0}+(h \pm w) U_{1}+U_{2}\right] .
$$

Rewriting and setting the equations of the two magnets equal we get a second order equation in $w$

$$
\begin{aligned}
& \left(V_{0}-\chi U_{0}\right) w^{2}+\left(2 h V_{0}+V_{1}+2 \chi h U_{0}+\chi U_{1}\right) w \\
& \quad+\left(h^{2} V_{0}+h V_{1}+V_{2}-\chi h^{2} U_{0}-\chi h U_{1}-\chi U_{2}\right)=0
\end{aligned}
$$

Here $V_{i}$ are the coefficients from Eq. (25) for the quadrupole upstream of the IP. Having determined $w$, Eq. (27) then gives $\beta_{w}$ and the average $\beta_{w}$ found for the two magnets is used to calculate $\beta^{*}$ as before using Eq. (21).

\section{Fringe fields}

Equation (3) assumes modulation of a thin quadrupole at a location with a single value of $\beta$. Sections II B and IIC assume this to correspond to the average $\beta$ function in a hard edge quadrupole. In practice, magnets also have fringe fields at the edges which scale with the quadrupole modulation but are not taken into account in the above sections. To estimate the error in tune shift due to this approximation, the magnet can be divided into many quadrupole slices, see Fig. 4 . The tune shift from modulating a single hard edge magnet can then be compared to the combined tune shift resulting from the modulation of all the magnet slices with the same total integrated field strength.

Each quadrupole slice is itself a hard edge magnet and the tune shift is given by Eq. (3) with $\beta$ being the average $\beta$ function inside the slice. The average of $\beta$ in slice number $i$ is given by Eq. (11)

$$
\begin{aligned}
\bar{\beta}_{i} & =u_{0, i} \beta_{i}-u_{1, i} \alpha_{i}+u_{2, i} \gamma_{i} \\
& =\left(\begin{array}{lll}
u_{0, i} & -u_{1, i} & u_{2, i}
\end{array}\right)\left(\begin{array}{c}
\beta_{i} \\
\alpha_{i} \\
\gamma_{i}
\end{array}\right) \\
& =\boldsymbol{A}_{i}\left(\begin{array}{c}
\beta_{i} \\
\alpha_{i} \\
\gamma_{i}
\end{array}\right) .
\end{aligned}
$$

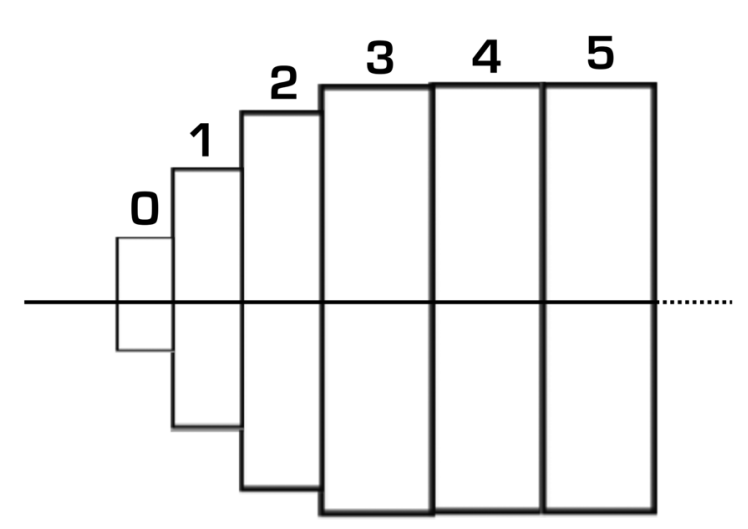

FIG. 4. Illustration of several hard edge quadrupole magnets, together modeling a quadrupole with varying field. The slices are numbered, $\beta_{0}, \alpha_{0}$, and $\gamma_{0}$ corresponds to the beta function at the entrance of slice 0 . 
$\boldsymbol{A}_{i}$ is here a $1 \times 3$ matrix, while $\beta_{i}, \alpha_{i}$ and $\gamma_{i}$ are the optical functions at the edge of the slice, see Fig. 4. Denoting the $3 \times 3$ transfer matrix though quadrupole slice $i$ by $\boldsymbol{T}_{i}$, the optical functions at the edge of slice $i+1$ are given by

$$
\left(\begin{array}{c}
\beta_{i+1} \\
\alpha_{i+1} \\
\gamma_{i+1}
\end{array}\right)=\boldsymbol{T}_{i}\left(\begin{array}{c}
\beta_{i} \\
\alpha_{i} \\
\gamma_{i}
\end{array}\right)=\prod_{j=0}^{i}\left(\boldsymbol{T}_{j}\right)\left(\begin{array}{c}
\beta_{0} \\
\alpha_{0} \\
\gamma_{0}
\end{array}\right),
$$

with $\beta_{0}, \alpha_{0}$, and $\gamma_{0}$ the optical functions at the start of the first slice. The average $\beta$ function of slice $i$ follows using Eq. (29)

$$
\bar{\beta}_{i}=\boldsymbol{A}_{i}\left(\begin{array}{c}
\beta_{i} \\
\alpha_{i} \\
\gamma_{i}
\end{array}\right)=\boldsymbol{A}_{i} \prod_{j=0}^{i-1}\left(\boldsymbol{T}_{j}\right)\left(\begin{array}{c}
\beta_{0} \\
\alpha_{0} \\
\gamma_{0}
\end{array}\right) .
$$

Approximating $\beta_{0} \Delta K L \approx \sum_{i=0}^{N-1} \bar{\beta}_{i} \Delta K L_{i}$ in Eq. (3), where $N$ is the number of slices, gives

$$
\begin{aligned}
& \cos [2 \pi(Q+\Delta Q)] \\
& =\cos (2 \pi Q) \pm \frac{\sin (2 \pi Q)}{2} \sum_{i=0}^{N-1} \Delta K L_{i} \boldsymbol{A}_{i} \prod_{j=0}^{i-1}\left(\boldsymbol{T}_{j}\right)\left(\begin{array}{c}
\beta_{0} \\
\alpha_{0} \\
\gamma_{0}
\end{array}\right)
\end{aligned}
$$

Assuming all slices are modulated by the same relative amount $\delta k$, Eq. (32) can be simplified by defining

$$
\boldsymbol{B}=\sum_{i=0}^{N-1} K L_{i} \boldsymbol{A}_{i} \prod_{j=0}^{i-1} \boldsymbol{T}_{j}
$$

which can be found given the strength and length of all the slices. Note that the change in integrated strength $\Delta K L_{i}$ here is exchanged with the total integrated strength $K L_{i}$, and that $\Delta K L_{i}=\delta k K L_{i}$, resulting in

$$
\Delta Q= \pm \frac{1}{2 \pi} \cos ^{-1}\left[\cos (2 \pi Q) \pm \frac{\sin (2 \pi Q)}{2} \delta k \boldsymbol{B}\left(\begin{array}{c}
\beta_{0} \\
\alpha_{0} \\
\gamma_{0}
\end{array}\right)\right]-Q
$$

\section{SIMULATIONS}

\section{A. SAD model}

To simulate $K$ modulation measurements in SuperKEKB the computer code Strategic Accelerator Design (SAD) has been used [19]. Lattices include detailed representations of the interaction region (IR) that includes fringe fields from the final focusing quadrupoles, detector solenoid, compensation solenoids and corrector magnets [20]. The fields from these elements have been simulated and divided into $1 \mathrm{~cm}$ slices that are interleaved throughout the IR model. Figure 5 shows the quadrupole fields near the IP in LER (above) and HER (below). As seen in the figure, there are fields leaking into the electron orbit in HER from the final focusing magnets in LER. When measuring $\beta^{*}$ in HER, it is therefore necessary to propagate the $\beta$ function past these fields using the method described in Sec. II C.

$K$ modulation is simulated by changing the integrated strength of all slices in the IR corresponding to the modulated magnet by the same relative amount. The resulting change in tune is then calculated by SAD, and is used to estimate $\beta^{*}$. The calculated value can then be compared with the value given by SAD, which takes into
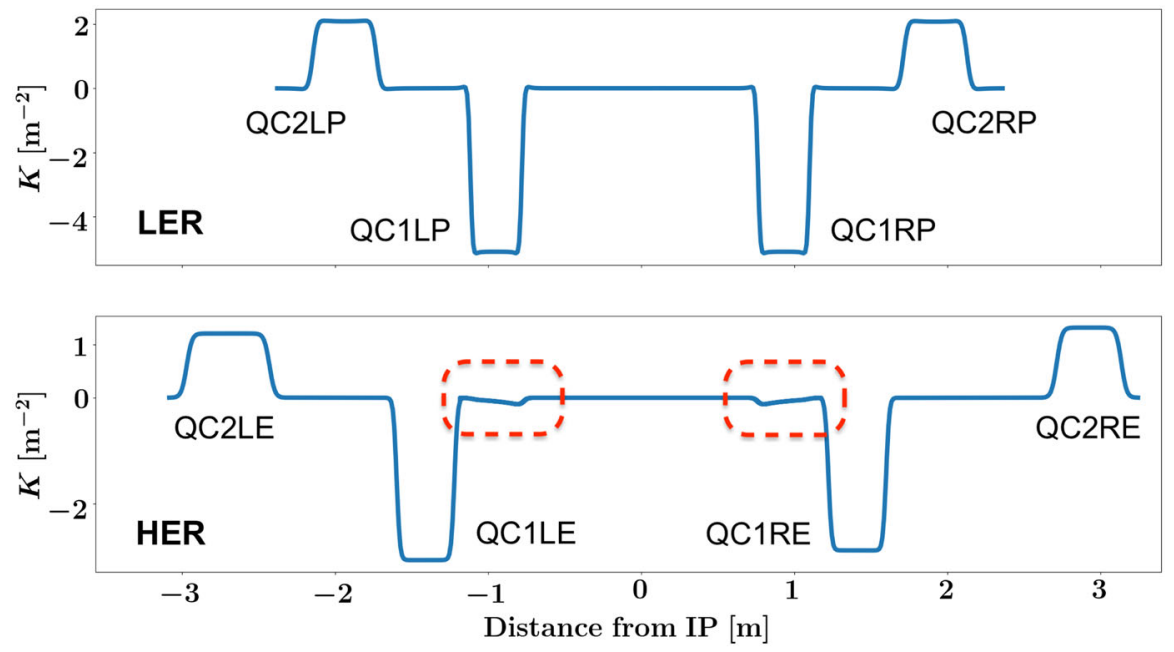

FIG. 5. Quadrupole fields in the orbits through the IR of LER (above) and HER (below). The names of the magnets are indicated. Fields from the inner quadrupoles in LER that leak into the HER orbit are pointed out with red rectangles. The focusing system is nearly symmetric, but notice slightly different lengths and placements of horizontally focusing magnets in HER. 
TABLE III. Uncertainties used for estimating error of $\beta^{*}$ measurements. $\Delta K$ is the uncertainty in the integrated strength of the modulated quadrupoles, while $\Delta K^{\dagger}$ is the uncertainty in the integrated strength of the quadrupole fields from LER present in the HER orbit. The tune uncertainty is for normal turn by turn measurements, while a gated turn by turn measurement for a pilot bunch can be used to get a tune uncertainty of $2 \times 10^{-5}$. For the other parameters typical values have been assumed. The gated turn by turn tune measurement could improve the accuracy, but was not used for the data presented in this paper.

\begin{tabular}{lc}
\hline \hline Parameter & Uncertainty \\
\hline$\Delta L^{*}[\mathrm{~mm}]$ & 1 \\
$\Delta h[\mathrm{~mm}]$ & 1 \\
$\Delta Q$ & $2 \times 10^{-4}$ \\
$\Delta K[$ relative $]$ & $10^{-3}$ \\
$\Delta K^{\dagger}$ [relative $]$ & $10^{-2}$ \\
\hline \hline
\end{tabular}

account the magnet fringe fields, coupling, and the changing optical functions.

\section{B. Simulating errors}

Estimating the uncertainty of the measurement is done by setting uncertainties on the values used to calculate $\beta^{*}$, shown in Table III. Calculating $\beta^{*}$ from the correct values of the parameters gives the measured value. The uncertainty is estimated by finding the spread of $\beta^{*}$ when doing the measurement 10000 times with the model parameters drawn from normal distributions with standard deviations equal to the relevant uncertainties.

\section{Results from simulations}

First simulations are done without any measurement uncertainty to validate the approximations above. Figure 6 shows the deviation of simulated measurements from the actual value for different values of $\beta_{y}^{*}$ ranging from $3 \mathrm{~mm}$ to $90 \mu \mathrm{m}$ in LER. For all the cases the deviation is less than $0.1 \%$. As $\beta_{y}^{*}$ decreases toward $90 \mu \mathrm{m}$ there is a sharp increase in the deviation. There are several assumptions

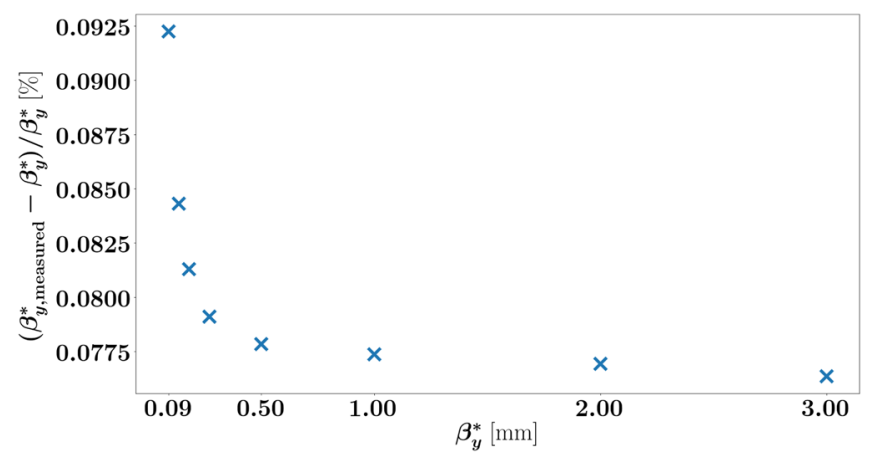

FIG. 6. Deviation of the calculated $\beta_{y}^{*}$ as a function of $\beta_{y}^{*}$ in LER, found from simulations using SAD in the absence of uncertainties.

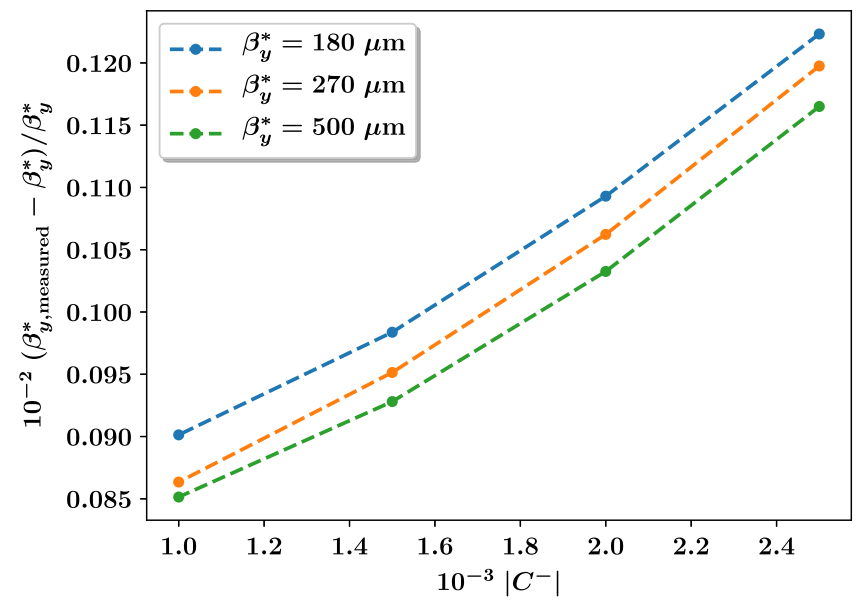

FIG. 7. Deviations in the calculated $\beta_{y}^{*}$ for different values of coupling strength in LER. The simulations are done for $\beta_{y}^{*}$ values of $500 \mu \mathrm{m}, 270 \mu \mathrm{m}$ and $180 \mu \mathrm{m}$. The quadrupole modulations are done such that the tune shifts away from the difference coupling resonance. The tune separation is 0.04 .

used that could contribute to the deviation of the measured value. First, in Eq. (27) it is assumed that $\beta^{*} \ll L^{*}$. Even for $\beta_{y}^{*}=3 \mathrm{~mm}$, a factor 10 above nominal, this term contributes less than $0.005 \%$ and it is therefore insignificant. It is also assumed that there are no orbit changes, and therefore no changes in tune from sextupoles in the ring. The orbit deviation as calculated by SAD affects the measured $\beta_{y}^{*}$ by less than $0.002 \%$, which does not account for the observed deviation.

The method further assumes that the horizontal and vertical planes are decoupled. This is true for the lattice used in SAD, where the coupling has been corrected, but for the real machine there will always be some amount of coupling. In this case the impact of the coupling can be reduced by modulating the quadrupoles such that the tune is shifted away from the difference coupling resonance. By adjusting skew quadrupole fields in the ring, the coupling

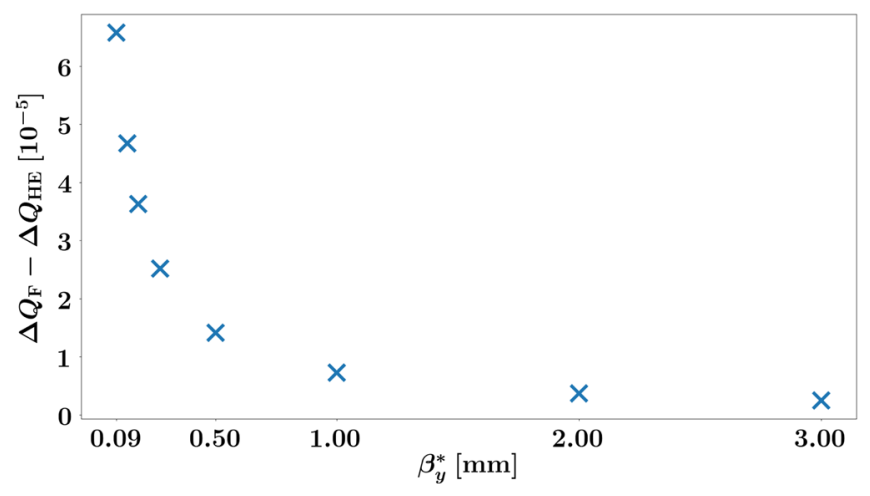

FIG. 8. Comparison of tune shifts for different $\beta_{y}^{*}$ calculated using Eq. (3) and taking into account the fringes by using Eq. (34). The calculations are based on the values for the LER magnet QC1LP, with $\Delta K L / K L=2 \times 10^{-5}$. 


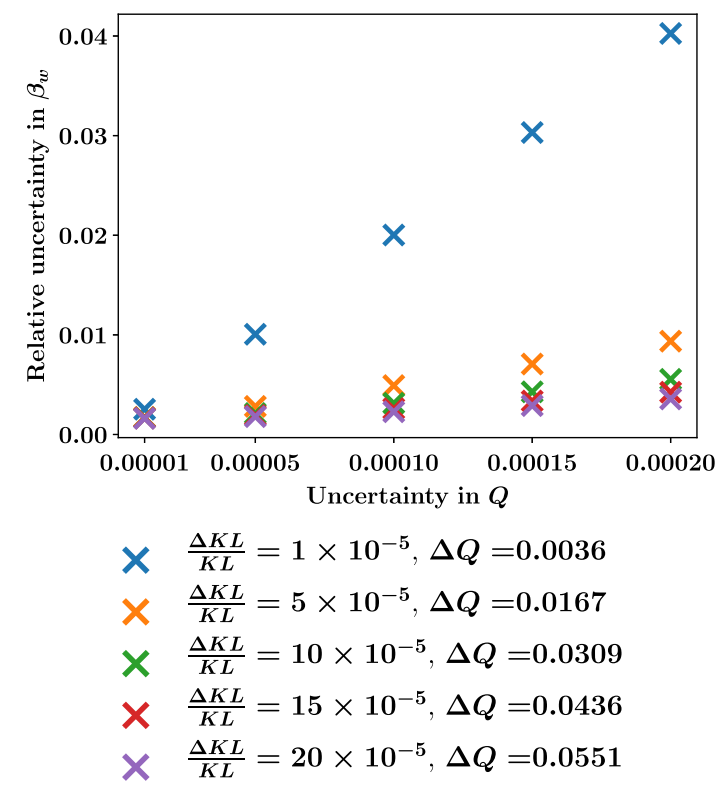

FIG. 9. Uncertainty in the $\beta_{w}$ measurement in LER for different uncertainties in the tune measurement, as well as for different values of modulation strength $\Delta K L$. Simulated for nominal $\beta_{y}^{*}=270 \mu \mathrm{m}$.

strength in the simulations has been varied and the corresponding deviations of the calculated $\beta_{y}^{*}$ are plotted in Fig. 7. Increasing coupling leads to larger deviations, as does smaller $\beta_{y}^{*}$. Aiming to achieve a nominal coupling below $\left|C^{-}\right|=2 \times 10^{-3}$ this gives a deviation below $0.11 \%$ [21,22]. In [10] a similar increase in deviations

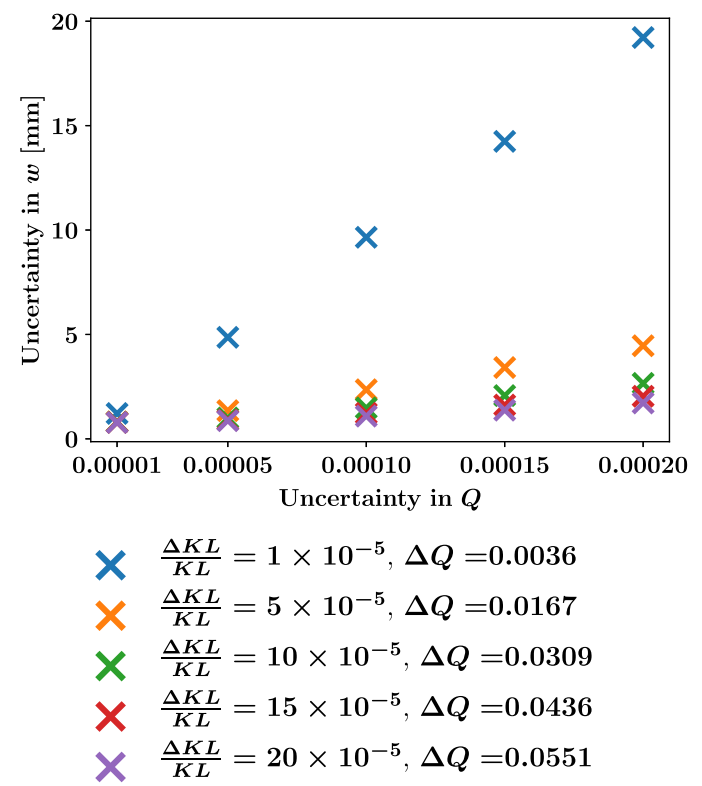

FIG. 10. Uncertainty in the $w$ measurement in LER for different uncertainties in the tune measurement, as well as for different values of modulation strength $\Delta K L$. Simulated for nominal $\beta_{y}^{*}=270 \mu \mathrm{m}$.
TABLE IV. Working point and $\beta^{*}$ during measurements in HER. The measurements were done on 5th of June 2018, during phase 2 of SuperKEKB commissioning.

\begin{tabular}{lc}
\hline \hline Parameter & Value \\
\hline$\beta_{y}^{*}[\mathrm{~mm}]$ & 3.0 \\
$\beta_{x}^{*}[\mathrm{~mm}]$ & 100 \\
$Q_{y}$ & 43.604 \\
$Q_{x}$ & 45.546 \\
\hline \hline
\end{tabular}
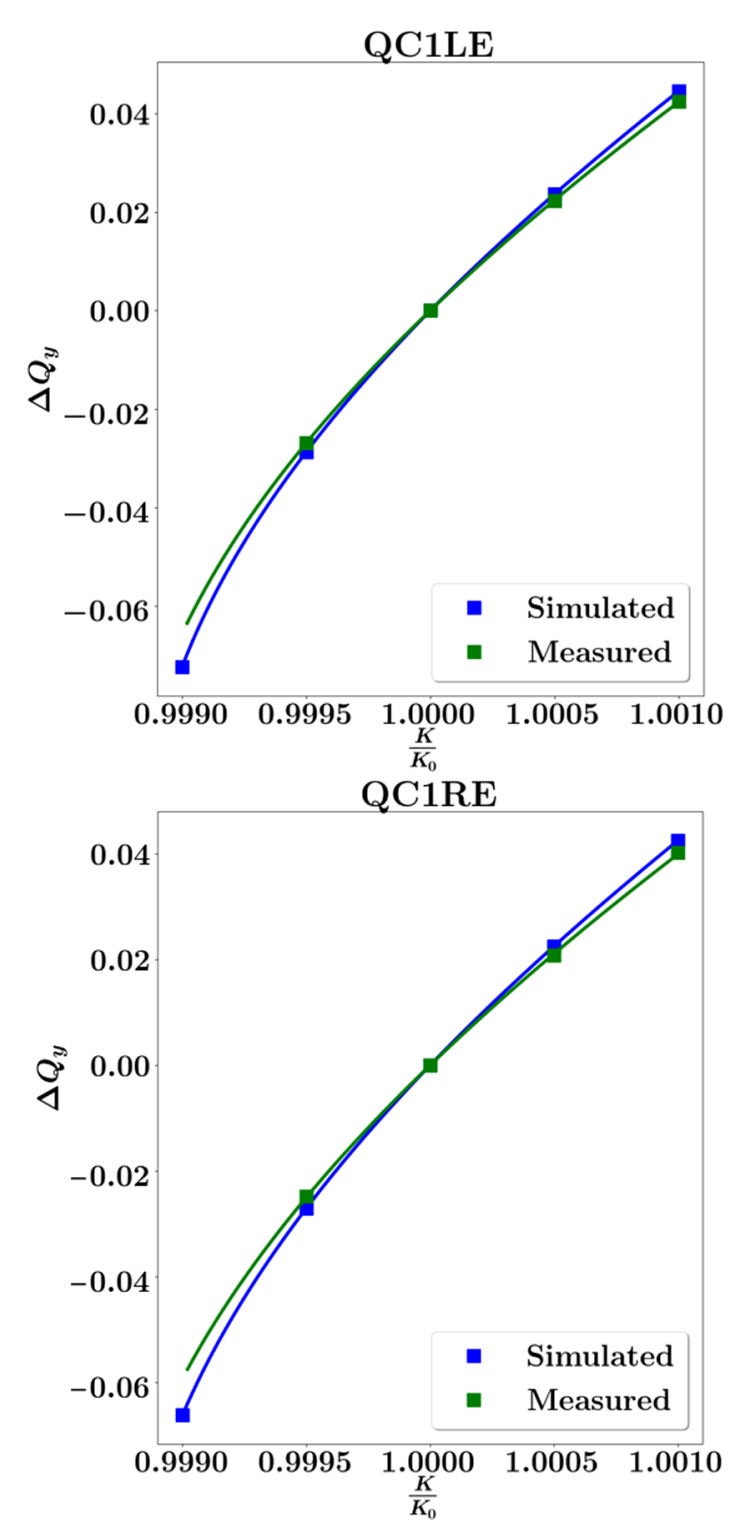

FIG. 11. Simulated and measured tune shift when modulating the inner quadrupoles in HER. The simulations are done using the design optics with $\beta_{y}^{*}=3 \mathrm{~mm}$. The uncertainty in the recorded tune measurements are $4 \times 10^{-4}$ for the modulation of the QC1LE magnet and $2 \times 10^{-4}$ for the modulation of the QC1RE magnet. The lines have been made by fitting the average $\beta$ function and using Eq. (3). The integrated strength of the magnets during the measurements were $K L_{0}=-1.15 \mathrm{~m}^{-1}$ for QC1LE and $K L_{0}=-1.09 \mathrm{~m}^{-1}$ for QC1RE. 
of calculated $\beta^{*}$ is found to stem from rotations in the modulated quadrupoles. The IR quadrupoles in SuperKEKB include small skew field components by design, which contribute to the observed deviations. Finally, Eq. (22) assumes that the modulated quadrupole magnet has a hard edge field profile. Using Eq. (34) and Eq. (3), the tune shift from modulating the QC1LP quadrupole in LER is calculated using both a hard edge model of the magnet and the sliced SAD model that include the fringe fields. The difference between these two values is shown as a function of $\beta_{y}^{*}$ in Fig. 8, and resembles the deviation in $\beta_{y}^{*}$ in Fig. 6. However, the exact value of the difference in tune shift is very dependent on the length of the hard edge model, varying between $10^{-5}$ and $10^{-4}$ for an uncertainty of $5 \mathrm{~mm}$ in $w$ at $\beta_{y}^{*}=90 \mu \mathrm{m}$.

The uncertainties in Table III are used to perform Monte Carlo simulations for the nominal LER lattice. The rms of the resulting $\beta_{w}$ and $w$ distributions are shown in Figs. 9 and 10, respectively. The large uncertainty in $w$ arises mainly from the uncertainty in the tune measurement and the error in the longitudinal placement of the magnets, assumed to be on the order of $1 \mathrm{~mm}$. From Eq. (21) it is clear that an uncertainty in $w$ of $1 \mathrm{~mm}$ results in a very large uncertainty in $\beta^{*}$ when $\beta^{*}$ is on the order of $1 \mathrm{~mm}$ and below. If $\beta_{w}=$ $0.3 \mathrm{~mm}$ and $w=1 \mathrm{~mm}, \beta^{*}=(0.3+1 / 0.3) \mathrm{mm}=3.6 \mathrm{~mm}$. Therefore, $K$ modulation is not suited for measuring $w$ in SuperKEKB. However, the estimated uncertainties for $\beta_{w}$ are promising, and can be combined with other methods for determining $w$, such as measuring the luminosity while varying the longitudinal position of the waist [23]. Note that $\beta_{w}$ is a function of $w$, however this is taken into account in the uncertainty estimates for $\beta_{w}$.

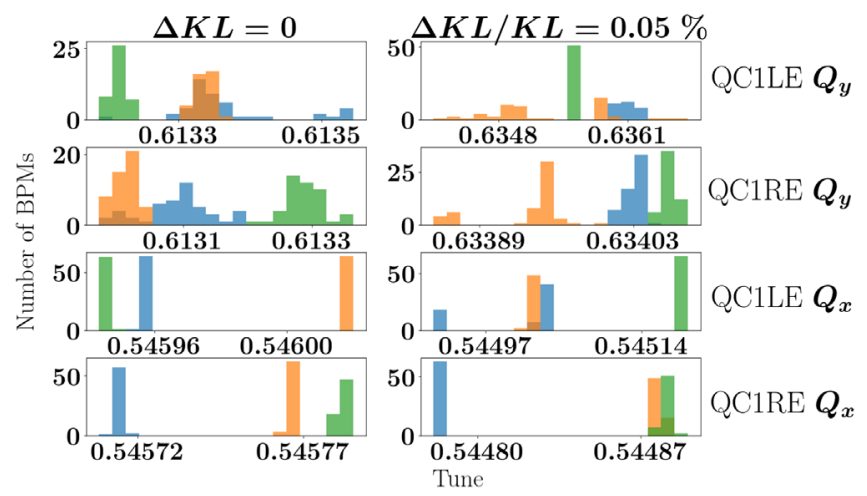

FIG. 12. Histograms showing the number of BPMs measuring a certain value of the tune. Three sets of turn by turn measurements were recorded for each setting of the two magnets QC1LE and QC1RE. The colors indicate the sequence of the measurements; blue being first, followed by orange, and green last. The histograms on the left column come from the reference measurements for the magnets with $\Delta K L=0$, while in the ones on the right column have $\Delta K L / K L=0.05 \%$. The integrated strength of the magnets during the measurements were $K L_{0}=-1.15 \mathrm{~m}^{-1}$ for QC1LE and $K L_{0}=-1.09 \mathrm{~m}^{-1}$ for QC1RE.

\section{EXPERIMENTAL MEASUREMENTS IN HER}

During phase 2 of the SuperKEKB commissioning process, $\mathrm{K}$ modulation measurements were taken in HER on the 5th of June 2018. One by one, all 4 quadrupoles in the IR were modulated by $\pm 0.05 \%$ and $+0.1 \%$. Since we are most interested in $\beta_{y}^{*}$ we will only look at the results from modulation of the quadrupoles closest to the IP, QC1LE and QC1RE. Following each modulation, three sets of turn by turn beam position monitor (BPM) readings were recorded, including three sets for each magnet without
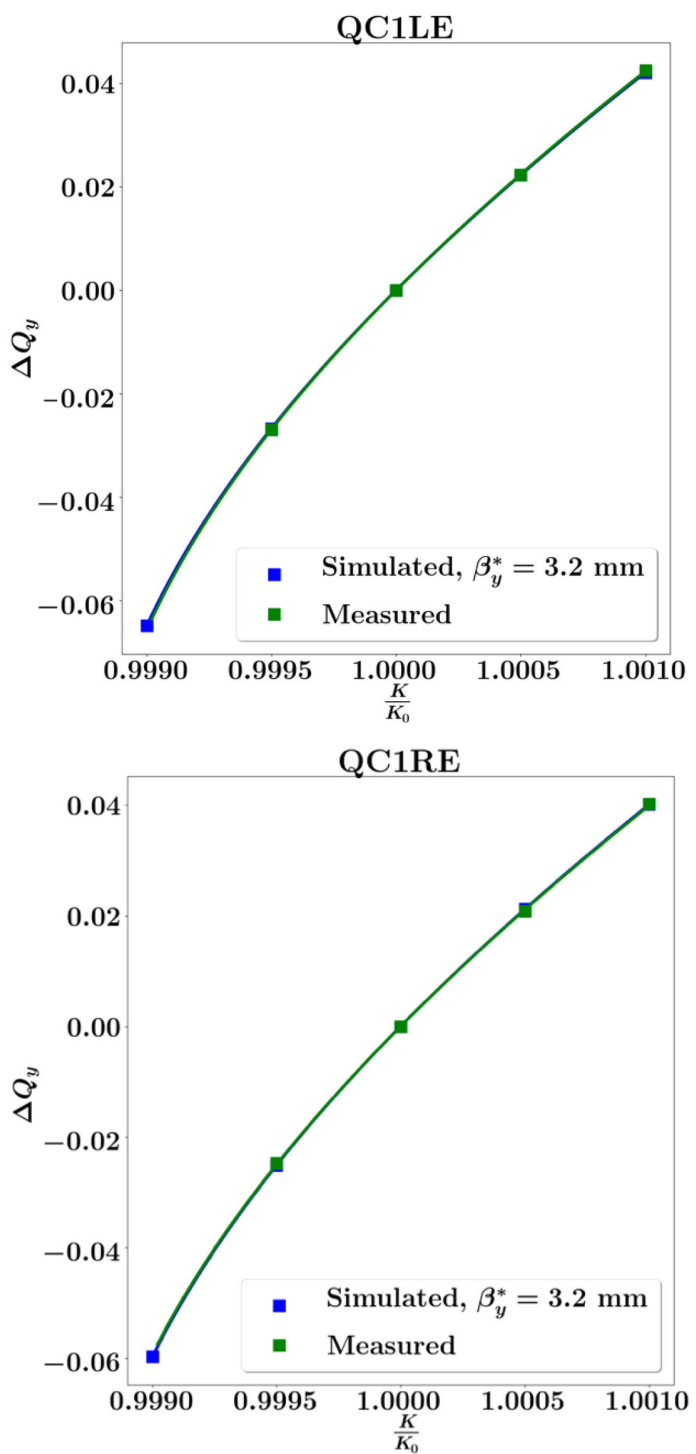

FIG. 13. Simulated and measured tune shift when modulating the inner quadrupoles in HER. The simulations are done using $\beta_{y}^{*}=3.2 \mathrm{~mm}$. The uncertainty in the recorded tune measurements are $4 \times 10^{-4}$ for the modulation of the QC1LE magnet and $2 \times 10^{-4}$ for the modulation of the QC1RE magnet. The lines have been made by fitting the average $\beta$ function and using Eq. (3). The integrated strength of the magnets during the measurements were $K L_{0}=-1.15 \mathrm{~m}^{-1}$ for QC1LE and $K L_{0}=$ $-1.09 \mathrm{~m}^{-1}$ for QC1RE. 
modulation as a reference. To ensure correct measurement of the tune shift, the tune feedback system was turned off. Settings during the measurements are displayed in Table IV and include $\beta_{y}^{*}=3 \mathrm{~mm}$.

In Fig. 11 the change in the vertical tune is plotted as a function of the relative change in quadrupole strength for the two vertically focusing magnets closest to the IP. Design optics in use during the measurements included $\beta_{y}^{*}=3 \mathrm{~mm}$, and the uncertainty in the recorded tune measurements are $4 \times 10^{-4}$ for the modulation of the QC1LE magnet and $2 \times 10^{-4}$ for the modulation of the QC1RE magnet. There was more tune jitter in the turn by turn measurements for QC1LE, which resulted in the higher uncertainty in the tune measurement. Figure 12 shows histograms of the tune measurements at all BPMs for several settings of the focusing quadrupoles, with different colors used for consecutive sets of turn by turn measurements, displaying a slight jitter in the tune but no visible tune drift. These tune uncertainties were combined with the estimated uncertainties in Table III and simulations were used to approximate the uncertainty in the measured values of $\beta_{w}$ and $w$. Figure 11 shows a small deviation from the simulated tune shifts using the design optics, and the measured $\beta$ function at the waist is $\beta_{w}=3.2 \mathrm{~mm}$ with an uncertainty of $0.8 \%$, not taking into account orbit shifts mentioned later. The waist itself was measured to be shifted by $w=-4.6 \mathrm{~mm} \pm 5 \mathrm{~mm}$ away from the IP. Figure 13 shows the measured tune shifts again, but this time the lattice used for simulation was matched to $\beta_{y}^{*}=3.2 \mathrm{~mm}$, indicating the close fit for all the measurements. It should be noted that the uncertainty in the tune measurement is a factor 100 smaller than the measured tune shifts, and it would therefore be possible to do the measurements with smaller quadrupole modulations.

Figure 14 displays the spectrum of the vertical position measurements from one of the BPMs in HER during the $K$ modulation measurements. The main frequency components correspond to the horizontal and vertical tunes, while

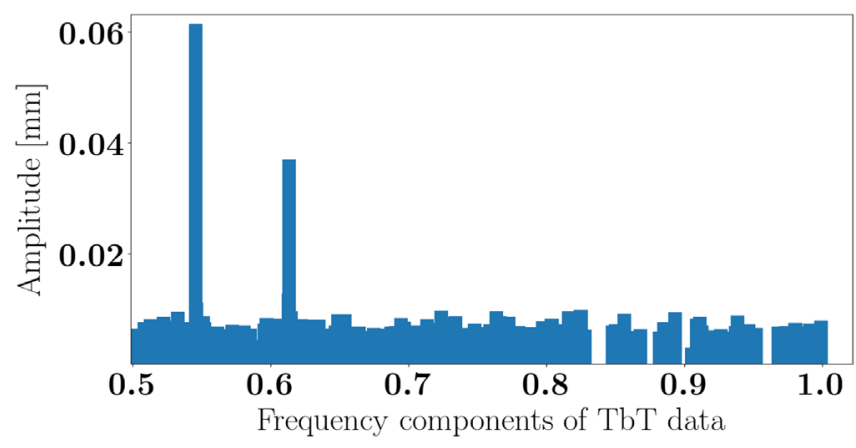

FIG. 14. The spectrum of the vertical position measurements from one BPM in HER during the $K$ modulation measurements. The main frequency components of the turn by turn (TbT) data correspond to the horizontal and vertical tunes in the ring shown in tune units.

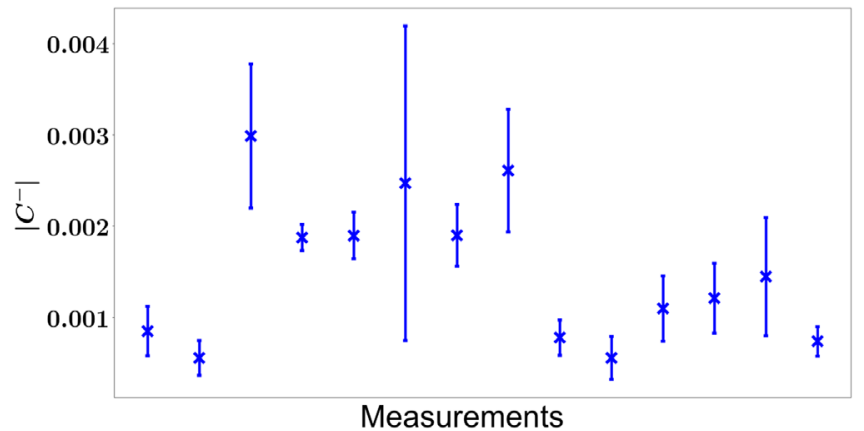

FIG. 15. Coupling in HER during the $K$ modulation measurements, estimated from the spectrum of turn by turn data from all BPMs [24]. When modulating the QC1LE magnet by $\Delta K L / K L=-0.05 \%$ the estimated coupling was a lot larger $\left|C^{-}\right|=0.07$, this measurement also shows the largest change in the orbit, see Fig. 16.

the relative amplitude of these frequency components in horizontal and vertical BPMs can be used to estimate coupling in the ring [24,25]. Coupling estimated from this method is shown in Fig. 15. When modulating the QC1LE magnet by $\triangle K L / K L=-0.05 \%$ a larger value of $\left|C^{-}\right|=0.07$ was measured, as well as larger changes in the measured orbits (see Fig. 16). Coupling starts to have an effect on the tune shifts once the horizontal and vertical tune separation approach the value of $\left|C^{-}\right|$. Figure 17 displays both the vertical and horizontal tunes during the measurements, and shows that the smallest separation of the tunes is 0.04 , when the magnets are modulated by
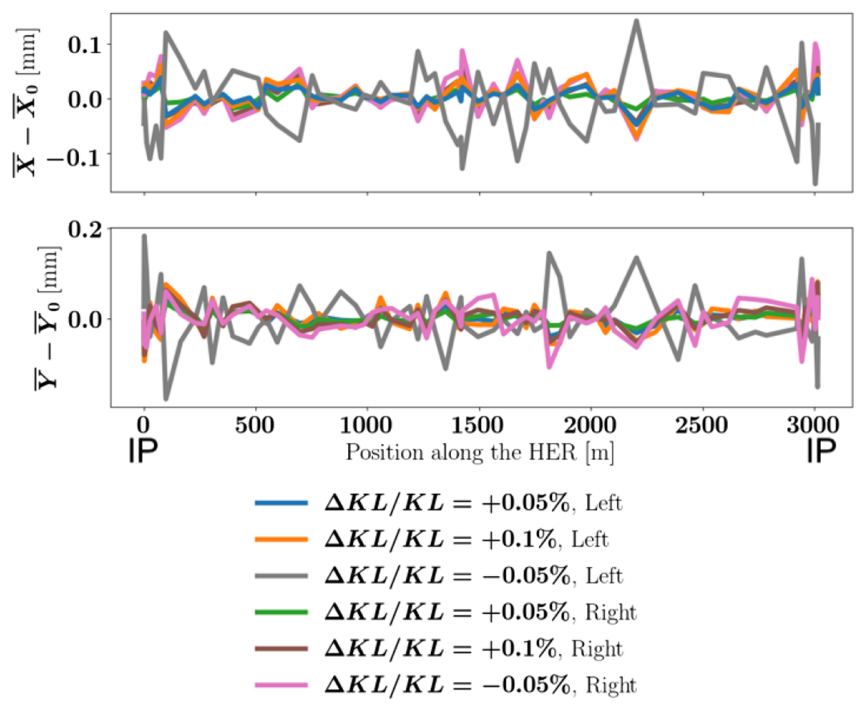

FIG. 16. Orbits calculated as the average of all position measurements from turn by turn data taken during the $K$ modulation measurements. The different colors indicate different modulations in the QC1LE and QC1RE magnets, left and right of the IP, respectively. The orbits are plotted as the deviation from the orbits found before modulating the magnets. 

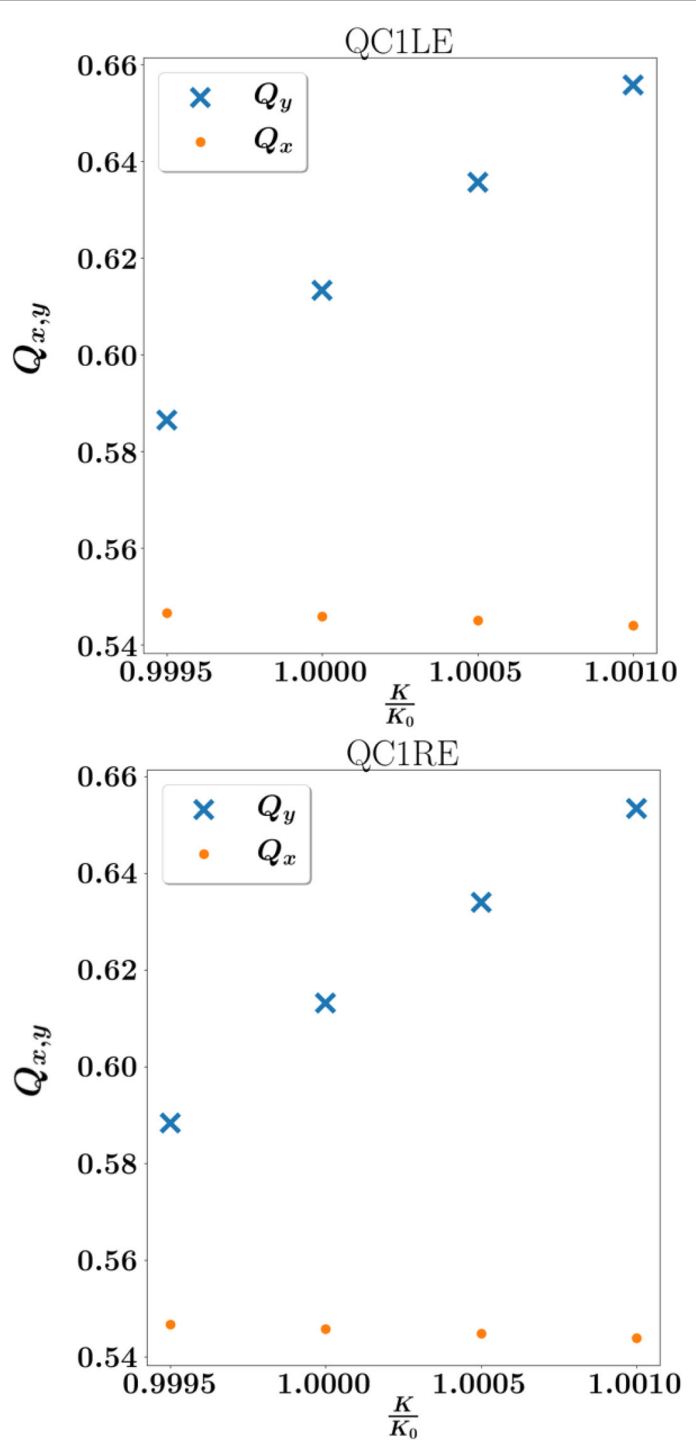

FIG. 17. Measured vertical and horizontal tunes while modulating the quadrupole magnets closest to the IP in HER, QC1LE and QC1RE. The smallest separation between the horizontal and vertical tunes is approximately 0.04 , which is 20 times larger than the estimated coupling strength during the measurements, $\left|C^{-}\right| \approx 0.002$, see Fig. 15. The integrated strength of the magnets during the measurements were $K L_{0}=-1.15 \mathrm{~m}^{-1}$ for QC1LE and $K L_{0}=-1.09 \mathrm{~m}^{-1}$ for QC1RE.

$\Delta K L / K L=-0.05 \%$. This is a factor 20 larger than the estimated coupling in the ring, $\left|C^{-}\right| \approx 0.002$. It should be mentioned that for the nominal parameters presented in Table I, the tune separation will be 0.04 in both HER and LER, equal to the smallest tune separation during the measurements in HER. Smaller separation than this can be avoided by only modulating up the strength of the quadrupole magnets, as this increases the vertical tune while decreasing the horizontal tune slightly.

An orbit displacement of $\delta x$ in a sextupole of strength $K_{2}$ and length $L_{s}$ gives a quadrupole kick and hence a tune shift that can be approximated by

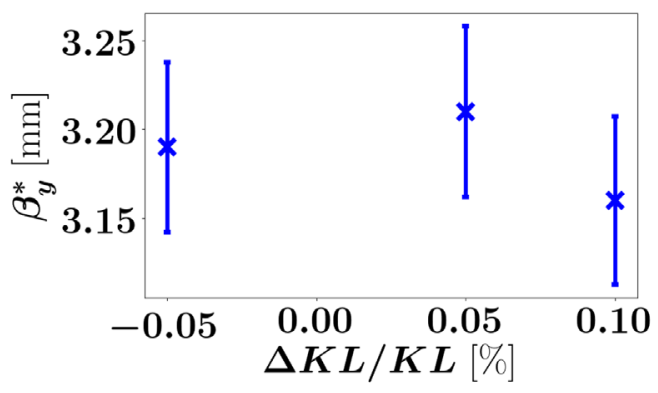

FIG. 18. Measured $\beta_{y}^{*}$ as a function of $\Delta K L$ for the $K$ modulation measurements in HER. Error bars of $1.5 \%$ are included.

$$
\Delta Q_{s} \approx \pm \frac{\beta_{s} K_{2} \delta x L_{s}}{4 \pi} .
$$

Using this formula, the observed orbit shift in HER results in a total tune shift of around $2 \times 10^{-4}$, which is the same as the uncertainty of the tune measurement. Including this as an increase of the tune measurement uncertainty gives a total uncertainty of the measured $\beta_{y}^{*}$ of $1.5 \%$. The feeddown from vertical orbit at the sextupoles to coupling is comparable to tune shift and negligible in this case. Figure 18 shows the final measured $\beta_{y}^{*}$ values for the different quadrupole modulations.

For the nominal SuperKEKB both the horizontal and vertical tunes will be closer to half integer, 0.53 and 0.57 , respectively. The relative deviation of the $\beta$ function from the design $\beta$ function when including errors in the quadrupole gradients $K$, called beta beating, can be shown to be proportional to $1 / \sin (2 \pi Q)$ as well as proportional to the value of the $\beta$ function at the error location [26]. Consequently, for a given quadrupole modulation the beta beating and orbit changes increase as the fractional part of the tune approaches 0.5 . However, since the $\beta$ function in the modulated quadrupole increases for smaller $\beta_{y}^{*}$, the strength of the quadrupole modulation can be decreased. $K$ modulation measurements should therefore be done again for smaller $\beta_{y}^{*}$ to assure that these effects do not interfere with the measurement. Additionally, the orbit and tune jitter could increase, in which case it is important to verify that the orbit correction system can keep the tune measurements stable enough for $K$ modulation.

\section{CONCLUSION AND FURTHER WORK}

In this paper we evaluate the prospects of using $K$ modulation to measure $\beta^{*}$ in SuperKEKB, covering first the needed theory and method. A new technique for finding the tune change from a modulated quadrupole is shown, allowing simple calculation of the second order tune shift due to $\Delta K$. Equation (15) can be used to estimate the size of this second order correction, and can be used also for other accelerators. Furthermore, an extension to the standard $K$ modulation method permits known fields to be located 
between the modulated quadrupole and the IP, generalizing the technique. This allows the method to be used in HER, where fields from magnets in LER are located between the final focusing quadrupoles and the IP. Also, a method to estimate the effects of fringe fields in the modulated magnets is developed and used to estimate the error from the hard edge magnet assumption.

$K$ modulation measurements in SuperKEKB are motivated by the need for accurate $\beta^{*}$ measurements for achieving the high luminosity goal of SuperKEKB, but also by the possibility of doing dedicated focus system studies that will give valuable input to future linear collider projects. From both simulations and experimental results from HER, it is found that $K$ modulation is suitable for measuring $\beta_{w}$ in SuperKEKB within $1 \%$, while the measurement of the position of the beam waist, $w$, is not accurate enough, varying by several $\mathrm{mm}$. One possibility is therefore to combine $K$ modulation with luminosity measurements while varying the position of the beam waist, a common technique used for positioning the beam waist at the IP. The main contributions to the uncertainty come from the uncertainty of the tune measurements, as well as in the uncertainty of the longitudinal placement of the modulated quadrupoles. The measurements from HER give $\beta_{y}^{*}=$ $3.2 \mathrm{~mm} \pm 1.5 \%$ for a design optics of $\beta_{y}^{*}=3.0 \mathrm{~mm}$, indicating a $7 \% \beta^{*}$ beating during phase 2 of commissioning. This $7 \%$ is compatible with the typical beta beating obtained after optics corrections in SuperKEKB [27]. Orbit shifts are found to affect the tune on the same order as the tune uncertainty, while second order terms in $\Delta K$ are found to contribute less than the uncertainty in the tune for $K$ modulation measurements in HER. The next steps are to streamline the measurements, validate the method in LER and test the method for optics closer to the nominal machine parameters with smaller $\beta_{y}^{*}$. Verifying the method for smaller $\beta_{y}^{*}$ is important as effects such as beta beating, orbit displacements, and tune jitter are affected by the larger $\beta$ functions in the final focus quadrupoles.

[1] P. Thrane, R. Tomás, and J. A. Støvneng, Measuring $\beta^{*}$ in SuperKEKB with $K$ modulation, Report No. CERNTHESIS-2018-300, 2018, https://cds.cern.ch/record/ $2652855 ? \ln =\mathrm{en}$.

[2] K. Akai, K. Furukawa, and H. Koiso, Superkekb collider, Nuclear Instrum. Methods Phys. Res., Sect. A 907, 188 (2018).

[3] T. Abe et al., Achievements of kekb, Prog. Theor. Exp. Phys. 2013, 03A001 (2013).

[4] F. Takasaki, The discovery of $\mathrm{cp}$ violation in b-meson decays, Proc. Jpn. Acad. Ser. B 88, 283 (2012).

[5] G. Casarosa, The Belle II experiment, J. Phys. Conf. Ser. 556, 012072 (2014).

[6] R. Tomás, M. Aiba, A. Franchi, and U. Iriso, Review of linear optics measurement and correction for charged particle accelerators, Phys. Rev. Accel. Beams 20, 054801 (2017).

[7] Y. Ohnishi, H. Sugimoto, A. Morita, H. Koiso, K. Oide, K. Ohmi, D. Zhou, Y. Funakoshi, N. Carmignani, S. M. Liuzzo, M. E. Biagini, M. Boscolo, and S. Guiducci, Optics measurements and corrections at the early commissioning of SuperKEKB, CERN-European Organization for Nuclear Research (2016), p. THPOR007, https:// cds.cern.ch/record/2268264/files/CERN-ACC-2017-0039 .pdf.

[8] M. Kuhn, V. Kain, A. Langner, and R. Tomás, First $\mathrm{k}$-modulation measurements in the LHC during run 2 , in Proceedings, 4th International Beam Instrumentation Conference, IBIC2015 (JACoW, Geneva, Switzerland, 2016).

[9] T. Persson, F. Carlier, J. C. de Portugal, A. G.-T. Valdivieso, A. Langner, E. H. Maclean, L. Malina, P. Skowronski, B. Salvant, R. Tomás, and A. C. G. Bonilla, LHC optics commissioning: A journey towards $1 \%$ optics control, Phys. Rev. Accel. Beams 20, 061002 (2017).

[10] F. Carlier and R. Tomás, Accuracy and feasibility of the $\beta^{*}$ measurement for LHC and high luminosity LHC using $\mathrm{k}$ modulation, Phys. Rev. Accel. Beams 20 (2017).

[11] The CLIC and CLICdp collaborations et al., Updated baseline for a staged Compact Linear Collider, arXiv: 1608.07537.

[12] G. Aarons et al. (ILC), ILC Reference Design Report Volume 1-Executive Summary, arXiv:0712.1950.

[13] H. G. Morales and R. T. Garca, Final-focus systems for multi-TeV linear colliders, Phys. Rev. Accel. Beams 17, 101001 (2014).

[14] S. Kuroda, ATF2 for final focus test beam for future linear colliders, Nucl. Part. Phys. Proc. 273-275, 225 (2016).

[15] V. A. Alexandrov et al., Results of final focus test beam, Conf. Proc. C950501, 2742 (1996).

[16] P. Thrane, Y. Funakoshi, Y. Ohnishi, K. Oide, H. Sugimoto, R. Tomás, and D. Zhou, Probing linear collider final focus systems in SuperKEKB, Tech. Reps. No. CERN-ACC2017-0052, CLIC-Note-1077 (CERN, Geneva, 2017), CERN-ACC-2017-0052, https://cds.cern.ch/record/ 2276026/files/CERN-ACC-2017-0052.pdf.

[17] F. Plassard, Optics optimization of longer $\mathrm{L}^{*}$ beam delivery system designs for CLIC and tuning of the ATF2 final focus system at ultra-low $\beta^{*}$ using octupoles, Ph.D. thesis, these de doctorat dirigée par Bambade, Philip et Tomás, Rogelio Physique des accélérateurs Paris Saclay 2018.

[18] M. Minty and F. Zimmermann, Measurement and Control of Charged Particle Beams (Springer, New York, 2003).

[19] Strategic Accelerator Design (SAD), http://acc-physics.kek .jp/SAD/.

[20] SuperKEKB Design Report, preliminary, https://kds.kek .jp/indico/event/15914/ [11.12.2018].

[21] H. Koiso, A. Morita, Y. Ohnishi, K. Oide, and H. Sugimoto, A Scheme for horizontal-vertical coupling correction at SuperKEKB, Proceedings of IPAC2012, New Orleans, Louisiana, USA (2012), p. 1203, http:// accelconf.web.cern.ch/AccelConf/IPAC2012/papers/ tuppc020.pdf. 
[22] Y. Ohnishi, Y. Funakoshi, H. Koiso, A. Morita, K. Oide, and H. Sugimoto, Optics correction and low emittance tuning at the Phase 1 commissioning of SuperKEKB, in Proc. of ICFA Advanced Beam Dynamics Workshop on High Luminosity Circular $e^{+} e^{-}$Colliders (eeFACT 16), Daresbury, UK, October 24-27, 2016, ICFA Advanced Beam Dynamics Workshop on High Luminosity Circular $\mathrm{e}^{+} \mathrm{e}^{-}$Colliders No. 58 (JACoW, Geneva, Switzerland, 2017) pp. 143-146, https://doi.org/10.18429/JACoWeeFACT2016-TUT3BH2.

[23] A. Morita, Status of SuperKEKB phase-2 commissioning, ICHEP (2018), https://indico.cern.ch/event/686555/ contributions/2962552/attachments/1681111/2700857/ ichep2018_KEK_A.Morita.pdf.

[24] A. Franchi, R. Tomás, and G. Vanbavinkhove, Computation of the Coupling Resonance Driving term f1001 and the coupling coefficient $\mathrm{C}$ from turn-by-turn single-BPM data, in Proceedings, 7th International Particle Accelerator Conference (IPAC 2016): Busan, Korea, 2016 (2010) CERN-BE-Note-2010-016, https://cds.cern.ch/record/ 1264111/files/CERN-BE-Note-2010-016.pdf.

[25] T. Persson and R. Tomás, Improved control of the betatron coupling in the large hadron collider, Phys. Rev. Accel. Beams 17, 051004 (2014).

[26] H. Wiedemann, Particle Accelerator Physics, 4th ed., Graduate Texts in Physics (Springer, New York, 2015).

[27] Y. Ohnishi, Y. Funakoshi, H. Koiso, A. Morita, K. Oide, and H. Sugimoto, Optics correction and low emittance tuning at the phase 1 commissioning of SuperKEKB, Proceedings, Proceedings of eeFACT2016: Daresbury, UK, 2016 (2016), p. 143, https://doi.org/http://accelconf .web.cern.ch/AccelConf/eefact2016/papers/tut3bh2.pdf. 